\title{
An experimental study on the evolution of modal damping with damage in carbon fiber laminates
}

Diogo Montalvão ${ }^{1}$, Dimitris Karanatsis ${ }^{1}$, António M.R. Ribeiro ${ }^{2}$, Joana Arina ${ }^{2}$ and Richard Baxter ${ }^{1}$

${ }^{1}$ School of Engineering and Technology, University of Hertfordshire, College Lane Campus, Hatfield, AL10 9AB, United Kingdom

${ }^{2}$ Mechanical Engineering Department, Instituto Superior Técnico, Technical University of Lisbon, Av. Rovisco Pais, 1049-001 Lisbon, Portugal

\section{Corresponding author}

Diogo Montalvão, School of Engineering and Technology, University of Hertfordshire, College Lane Campus, Hatfield, AL10 9AB, United Kingdom.

Email: d.montalvao@herts.ac.uk

\begin{abstract}
Many of the techniques developed to assess structural damage are based on Experimental Modal Analysis (EMA). This paper presents a study to extend the current understanding of how increasing damage in a Carbon Fiber Reinforced Plastic (CFRP) affects the modal damping factor of a laminated structure. Damage is introduced and quantified in terms of the dissipated energy. It is shown that there is a tendency for the overall damping to increase whereas there is a tendency for the overall stiffness to decrease. While these results are not novel, the former is quite relevant, since the authors are not aware of many other experimental studies on the evolution of the modal damping factor with damage in CFRPs. At the same time, a modal-based damage location technique that combines both the natural frequencies and the modal damping
\end{abstract}


factors as damage sensitive features is discussed. The hypothesis that different damage morphologies on composite materials have different contributions to the damage features is drawn. The methods are illustrated with both numerical and experimental examples. One of the problems observed is that, although damping is consistently found to increase globally with damage, the determination of the individual changes of the modal damping factors is still very uncertain. This may be due to concurrent damage types being present at the same time, but most certainly due to uncertainties involved in the identification of the modal damping factors.

\section{Keywords}

Structural Health Monitoring; Damage Detection; Delamination; Natural frequency; Modal damping factor.

\section{Introduction}

Composite materials are being used in many modern structures, in applications as diverse as vehicles (cars and aircraft), buildings and sports equipment The Airbus A380 is $25 \%$ by weight composites including $23 \%$ carbon fiber reinforced polymer and $3 \%$ fiberglass reinforced aluminum ${ }^{1}$. The Boeing 787 Dreamliner is pushing the envelope with a total composite of $50 \%$ by weight, including the integration of an all composite fuselage, wings and tail.

However, composite materials are very different from metals with respect to their micromechanical interactions and their particular failure modes, which may be in the form of matrix cracking, fiber breakage or interlaminar delamination ${ }^{2,3,4}$. Furthermore, the extreme sensitivity of composite materials to impact loads constitutes a hindrance to 
its further utilization. In fact, in aeronautical structures, the components have to undergo low energy impacts caused by dropped tools or mishandling during assembly and maintenance; low to medium energy impacts caused in-service by foreign objects, such as hailstones, stones or birds; and high energy impacts caused by weapon projectiles in military planes ${ }^{4,5,6}$.

Impact damage is introduced only after the impact force reaches a certain threshold level. Once that threshold is reached, a crack will appear, propagate and reach the interfaces between the current and adjacent plies. High transverse shear stresses will grow and delaminations appear. On the opposite side of the impact, due to the development of high tensile forces, fiber breakage may occur as well ${ }^{6,7}$.

Interlaminar delamination greatly reduces the part's stiffness and maximum buckling load, which, in turn, influences the structural stability. Fiber breakage, on the other hand reduces the structural capability in general, both in terms of load capability and stiffness ${ }^{8}$.

Most traditional damage assessment methods are based on the fact that damage leads to appreciable reduction in the stiffness of a structural element ${ }^{9}$. Nevertheless, in structures made of composite materials several authors recommend using damping variation associated with the dissipated energy - to determine damage extent, since these tend to be more sensitive to damage than the stiffness variations, mainly when delamination is concerned. For instance, in the late 70's a formulation for damage detection, localization and quantification was already indicating that damping might suffer an increase with damage on CFRPs ${ }^{10}$. Based on the observation that modal damping is a parameter with higher sensitivity to internal delamination on CFRPs than the natural frequencies, a method was developed using a forward approach, in which the 
experimental and analytical damping deviations were correlated through a modified form of the Modal Assurance Criterion (MAC) ${ }^{11}$. In other work ${ }^{12}$, the Specific Damping Capacity (SDC) is defined and used for damage location in laminates. It was observed that the resonant frequency did allow detecting cracks in Glass Fiber Reinforced Plastic (GFRP) laminates while, for CFRPs, no detectable changes in the resonant frequencies could be found; however, even for CFRPs, high changes were found in the SDC. The energy dissipation in a CFRP is mostly induced by the interfacial slip across the delamination and the tendency for mutual penetration between the upper and lower surfaces in the delamination region ${ }^{13}$. Thus, since damping is an energy dissipation mechanism, it is reasonable to assume it may be used as a parameter for Structural Health Monitoring (SHM). In such a case, the material is locally non-uniform and damping may be expected to increase ${ }^{14}$, depending on the deformation shape. On the other hand, fiber breakage may also be present which will induce a natural frequency variation. For more severe damage levels, changes in natural frequencies will be marked and damping may even decrease ${ }^{15,16}$. For thin laminates and in real-life situations, fiber breakage is very likely to occur because of the bending stresses in the back side of the laminate. If one imagines that each fiber acts as a spring-damper system and that the breakage corresponds to the interruption of the spring-damper connection, it is reasonable to assume that a local decrease in both stiffness and damping may happen simultaneously. Furthermore, the debonding between two adjacent laminae will reduce the bending stiffness. This dichotomy - where damping can either increase or decrease depending on the damage type present - may have the adverse consequence of masking modal changes due to combined fiber breakage and delamination damage. This may well be one of the reasons for the modal damping factor to pose some problems in 
damage identification algorithms, along with the uncertainty associated to its identification.

Following previous works ${ }^{17,18}$, this paper presents a study to understand how increasing damage - quantified by the energy that is dissipated during its introduction - affects the global modal parameters, i.e., modal damping factors and natural frequencies. A modalbased damage location technique that combines both the natural frequencies and the modal damping factors as damage sensitive features is also discussed. One objective was to understand if the method, originally conceived for damage location in laminated composite plates (level 2 of Rytter's damage state classification system ${ }^{19}$ ), can be used to assess damage type or morphology (level 3 of Rytter's scale). The other objective was to show, using classical Experimental Modal Analysis techniques, that a correlation between the modal parameters evolution and damage can be established. There is a relationship between the dissipated energy and the modal parameters, which is shown, although investigation is still required to further understand the meaning of the correlation.

While the effect of damage on stiffness (frequency) is well appreciated, the authors are not aware of many other experimental studies that assess the evolution of the modal damping factors with increasing damage in CFRPs woven laminates. For example, there are works that relate the loss factor measured from the hysteresis loop during one cycle of vibration with damage ${ }^{20,21}$ for beams. Similarly, in other works, only the first mode is assessed, for example using the SDC and Finite Element Modeling updating ${ }^{22}$ or, more simply, the logarithmic decrement for a beam under free vibration ${ }^{23}$. In another study, the effects of different levels of impact damage on the modal damping factors of carbon fiber entangled sandwich beams is discussed ${ }^{24}$, suggesting that damping may be used 
instead of the natural frequency as a damage indicator for SHM. The present paper is a contribute to the understanding of how the modal damping factor, using Experimental Modal Analysis (EMA) techniques, can provide information about damage in CFRPs.

\section{Materials and Methods}

\section{Case-studies}

Below, a numerical example with two types of damage is described, using the Multiparameter Damage Indicator $(\mathrm{MuDI})^{18}$. This index for detecting the most likely location of damage in a CFRP laminate includes information from both the frequency and damping modal parameters.

We also discuss two experimental examples, in which damage is introduced at a single location and gradually increased. In these experimental examples, we can observe how the modal frequencies and damping factors change with the increase in damage.

\section{The Multi-parameter Damage Indicator (MuDI)}

The MuDI has been previously described ${ }^{18}$. It is the weighted combination of two other indexes, the Damping Damage Indicator (DaDI) and the Frequency Damage Indicator (FreDI). This index is based on the assumption that a delamination in a composite laminate may lead to changes both in the natural frequencies and in the modal damping factors. The relative difference between the healthy and damaged states of the modal damping values $\eta$ for mode $r$ is defined as ${ }^{17,18}$ : 


$$
\Delta \eta_{r}^{D}=\frac{\left|\eta_{r}^{D}-\eta_{r}\right|}{\eta_{r}} \times 100 \%
$$

where the superscript ${ }^{D}$ stands for damaged state and $\eta_{r}$ are the modal damping factors $(r=m \ldots n$, where $m$ and $n$ are, respectively, the first and last modes in the measured frequency range). The relative difference of the hysteretic damping factor when comparing the healthy and damaged states is obtained independently of the coordinates under consideration, since damping factors are global properties of the structure. However, when a discrepancy exists due to localized damage, a function must be used to relate this global variation to the local coordinates of the structure.

A generalized Plane Shape Function (PSF) is defined for mode $r$ at a discretisation node with coordinates $(i, j)$ as a function of the mode shape. Definition of a PSF when damping changes are concerned and for bi-dimensional structures was proposed elsewhere as ${ }^{17,18}$ :

$$
{ }_{\eta_{r}} P S F_{i j}={ }_{r} S_{x x i j}{ }_{r} \varepsilon_{x x i j}+{ }_{r} S_{y y i j}{ }^{r} \varepsilon_{y y i j}
$$

where $S$ stands for stress and $\varepsilon$ for strain.

It is assumed that the mode shapes will not suffer a considerable change when comparing the undamaged and damaged states of the structure. An index, called Damping Damage Indicator (DaDI), was defined as ${ }^{17,18}$ :

$$
D a D I_{i j}=\frac{\sum_{r=m}^{n}\left({ }_{\eta_{r}} P S F_{i j} \cdot \Delta \eta_{r}^{D}\right)}{\sum_{r=m}^{n} \eta_{r} P S F_{i j}}
$$

Applying equation (3) requires only the damping factor values for both the damaged and undamaged structure and the PSF that can be obtained with a Finite Element Model (FEM). As the modal damping factors are global properties of the structure, only one 
measurement coordinate is strictly required, independently of the structure dimension and geometry, although more can be used to increase the performance of the analysis.

The natural frequency and the damping factor may be considered complementary modal features to assess the damage location, either because delamination also corresponds in a loss of stiffness or because other damage mechanisms may be involved (e.g., fiber breakage). The relative difference between the healthy and damaged state of the natural frequency $\omega_{r}$ of mode $r$ may be defined as ${ }^{17,18}$ :

$$
\Delta \omega_{r}^{D}=\frac{\left|\omega_{r}^{D}-\omega_{r}\right|}{\omega_{r}} \times 100 \%
$$

The resonant frequencies are stiffness dependent. The strain, for a given load, is a measure of the flexibility of the structure, so it can be used as a PSF for the FreDI. When computing the FreDI the following form of the PSF was proposed elsewhere ${ }^{17,18}$ :

$$
{ }_{\omega_{r}} P S F_{i j}=\left|{ }_{r} \varepsilon_{x x i j}+{ }_{r} \varepsilon_{y y i j}\right|
$$

Then, in a similar way as for DaDI, an index, called the Frequency Damage Indicator (FreDI), was proposed as ${ }^{17,18}$ :

$$
F_{r e D I}=\frac{\sum_{r=m}^{n}\left({ }_{\omega_{r}} P S F_{i j} \cdot \Delta \omega_{r}^{D}\right)}{\sum_{r=m}^{n} \omega_{r} P S F_{i j}}
$$

Both DaDI and FreDI indexes for the damage location can be combined in a new index, introducing weighting functions $W_{f}$ and $W_{d}$ affecting both of them ${ }^{17,18}$ :

$$
M u D I_{i j}=W_{f} \cdot \frac{F e D I_{i j}}{\max \left|F r e D I_{i j}\right|}+W_{d} \cdot \frac{D a D I_{i j}}{\max \left|D a D I_{i j}\right|}
$$

This new index accounts for both the frequency and damping variations, as well as for the PSF mode shape based functions. Therefore, the index given by equation (7) is 
called Multi-parameter Damage Indicator (MuDI). However, one question arises: how are the weighting functions derived?

The proposed weighting functions' values are based on the dispersion of the variation of the modal frequencies and modal damping factors with respect to each mode shape. The relative standard deviations $\sigma_{\Delta \eta}$ and $\sigma_{\Delta \omega}$ for the set of values $\Delta \eta_{r}^{D}$ and $\Delta \omega_{r}^{D}$ respectively are determined. These standard deviations can be used as a global measure of how the modal damping factors and natural frequencies change with damage. Then, a frequency weighting is defined as ${ }^{17,18}$ :

$$
W_{f}=\frac{\sigma_{\Delta \omega}}{\sigma_{\Delta \eta}+\sigma_{\Delta \omega}}
$$

It is easier to treat the weighting functions as if they are complementary. So, by imposing the relationship $W_{f}+W_{d}=1$ the damping weighting function can also be calculated $^{17,18}$ :

$$
W_{d}=\frac{\sigma_{\Delta \eta}}{\sigma_{\Delta \eta}+\sigma_{\Delta \omega}}
$$

In practice, $\sigma_{\Delta \eta} \gg \sigma_{\Delta \omega}$ for most typical situations ${ }^{18,25}$, mostly due to the magnitude of the uncertainties involved during the modal identification process. Consequently, the direct application of equation (8) is useless because it would lead to $W_{f} \cong 0$ and $W_{d} \cong 1$, i.e., $M u D I_{i j} \cong D a D I_{i j}$. Equation (8) is therefore proposed to be re-written as ${ }^{17,18}$ :

$$
W_{f}=\frac{\kappa \cdot \sigma_{\Delta \omega}}{\sigma_{\Delta \eta}+\kappa \cdot \sigma_{\Delta \omega}}
$$

in which $K$ is a scale factor. While the identification of the natural frequencies is usually quite accurate, the same cannot be said for the damping factors. Both are 
determined by averaging over sets of data obtained during experimental testing. So, after the modal identification process is over, the obtained values for each of the natural frequencies $\omega_{r}$ and modal damping factors $\eta_{r}$ are in fact averaged values with corresponding relative standard deviations $\sigma_{\omega r}$ and $\sigma_{\eta r}$ for each mode. The damping factor is, in most structural applications in engineering, very small and hard to determine. If its value floats around an intermediate value, averaging is an easy tool available to estimate an approximate value of the damping factor. However, damping measurements often vary much more than estimations of the natural frequencies. As a consequence, for any mode $r$, the relation between the standard deviations of the numerator of expressions (1) and (4) was proposed as a metric for the scale factor $\kappa^{17}$, 18 :

$$
\kappa=\frac{\sum_{r=m}^{n} \sqrt{\sigma_{\eta r}{ }^{2}+\sigma_{\eta r}^{D^{2}}}}{\sum_{r=m}^{n} \sqrt{\sigma_{\omega r}{ }^{2}+\sigma_{\omega r}^{D^{2}}}}
$$

These quantities are determined automatically with a modal identification software package called BETAlab $^{25}$ that makes use of the Characteristic Response Function ${ }^{26}$.

\section{Numerical examples}

\section{Specimen's modeling and damage characterization}

Numerical examples were built in ANSYS ${ }^{\circledR}$ using a quasi-isotropic CFRP rectangular thin plate specimen with a $[0 / 45 /-45 / 90]_{S}$ layup and approximate dimensions of $392 \times 242 \times 2.1 \mathrm{~mm}$. Among other reasons, the dimensions of these specimens were chosen so that the ratio of the side lengths is not an integer to avoid double-modes. Each layer is considered to be a woven prepreg constituted by Hexcel® G803 3K 5H satin carbon 
fiber impregnated in HexPly® 200 phenolique matrix (200/40\%/G803). These laminates are in fact used in real structures, for instance, in some parts of the Lockheed Martin C-130 airplane.

The mechanical properties of the specimens' constituent materials described earlier were determined experimentally, namely the Young's and shear moduli, specific weight, Poisson's ratios and damping factors. Several different approaches, from theoretical to experimental, were followed ${ }^{19}$. As far as damping is concerned, the peculiarities of its measurement in composite materials have been addressed elsewhere $^{27}$. In this specific case, tensile tests showed that the composite layup being studied could in fact be approximated by a quasi-isotropic homogeneous material. Accordingly, the following properties were estimated: $E=44.64 \mathrm{GPa}, v=0.33, \rho=1508$ $\mathrm{kg} / \mathrm{m}^{3}, \eta=0.0027$.

To relate this simulation more closely to a real experimental setup, the influence of the excitation and measurement devices was also considered. In this simulation the force transducer was modeled with a theoretical active mass of $18 \mathrm{~g}$ (out-of-plane direction), total mass of $21 \mathrm{~g}$ and (estimated) mass moments of inertia $I_{x}=I_{y}=1.33 \times 10-6 \mathrm{kgm}^{2}$ and $\mathrm{I}_{\mathrm{Z}}=8 \times 10^{-7} \mathrm{kgm}^{2}$. The force transducer was modeled as a zero-thickness distributed mass, with concentrated mass moments of inertia, occupying an approximately square area of $2 \times 2$ elements. At this same point, a spring with $500 \mathrm{~N} / \mathrm{m}$ stiffness, modeled with a COMBIN14 line element, is attached, in order to simulate some of the constraint effects introduced by the push-rod and shaker. It was assumed that the responses, necessary to determine the Frequency Response Functions (FRFs), were measured with a contactless transducer, e.g., a LASER Doppler Vibrometer (LDV). 


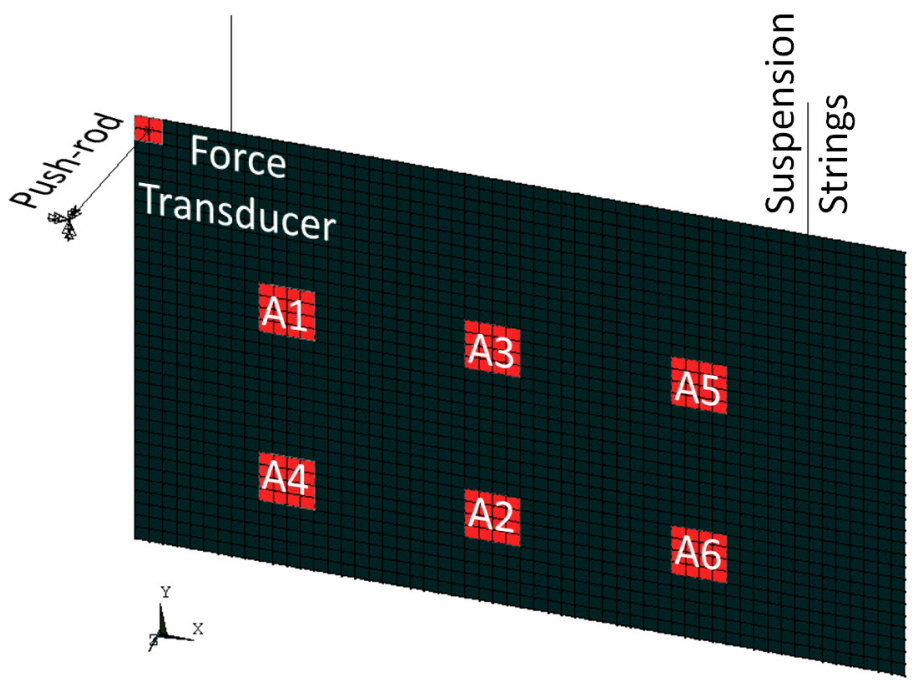

Figure 1. FE model with highlighted force transducer and damage locations.

For the damage location examples, 6 regions were considered (designated A1 to A6). The full model is illustrated in figure 1. Each of the 6 damaged locations is a square region modeled with $4 \times 4$ (16) elements, each region corresponding to an area of less than $1 \%$ of the total area of the plate. Damage was characterized by a local variation of either the damping factor ${ }^{1}$ or the Young's modulus by setting different values to each of the corresponding elements, providing a global variation in both the modal damping factors and natural frequencies. The following damage scenarios were considered:

100x damping factor increase ${ }^{2}$ in each of regions A1 to A6, individually;

10x damping factor increase in region A1 only;

10x Young's modulus reduction in region A1 only.

The type of damping used is referred by ANSYS ${ }^{\circledR}$ as Frequency Independent.

This increase was chosen in order to obtain modal damping factor (global) variations of the same order of magnitude of some experimental results previously obtained ${ }^{17,18}$. 
Region A1 was used as a reference region with more damage scenarios considered. Regions A2 to A6 were used to increase the sample size in the assessment of the global effects of damage on the modal parameters.

\section{The weighting functions and damage type}

A total of 18 mode shapes was used in this study, in the range of 0 to $800 \mathrm{~Hz}$. An estimate for the location of damage is shown by the MuDI index values along the plate. Areas with a larger index value are more likely to be damage locations. Results for a 10x local damping increase and a 10x local Young's modulus reduction are shown in figures 2 and 3, respectively. In figure 2 the correct location of damage is indicated using the DaDI whereas in figure $\mathbf{3}$ it is exactly the opposite that is happening, it is the FreDI index that locates the damage more accurately.

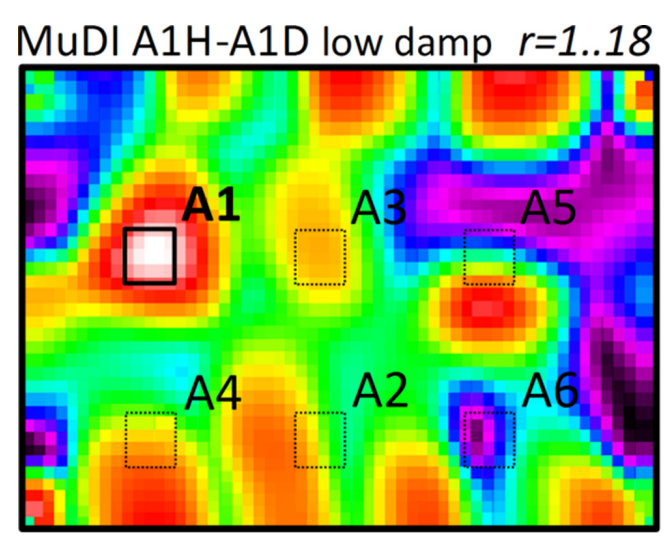

Lower Probability

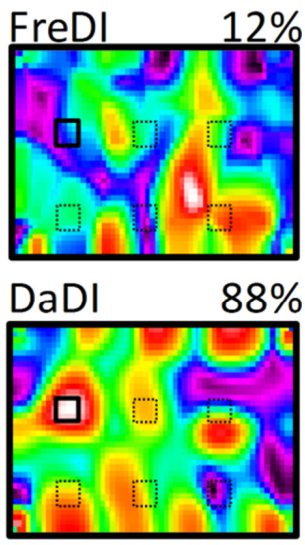

Higher Probability

Figure 2. Numerical results and weighting coefficients for a damping increase of 10x in region A1. 


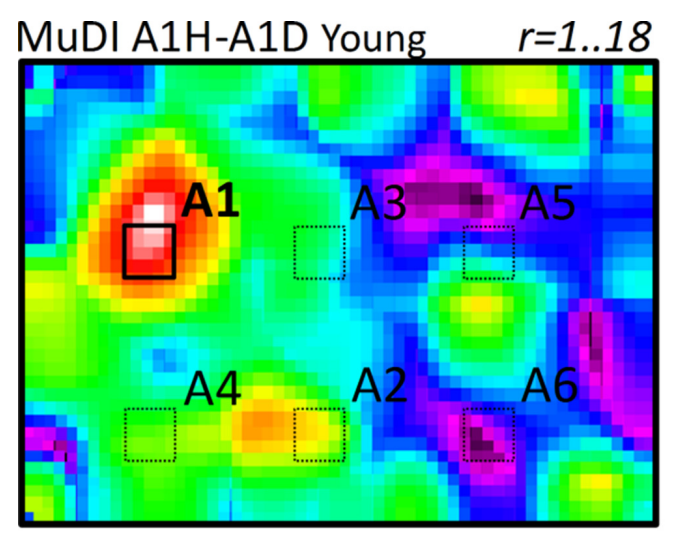

Lower Probability

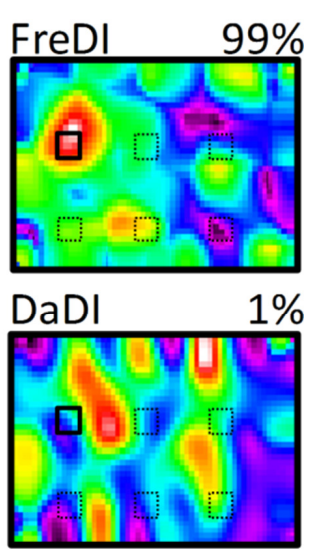

Higher Probability

Figure 3. Numerical results and weighting coefficients for a reduction in the Young's modulus of $10 \mathrm{x}$ in region $\mathrm{A} 1$.

The important role that weighting functions $W_{f}$ and $W_{d}$ - equations (9), (10) and (11) play the damage location procedure can also be observed. In fact, the weighting functions can also provide hints as to the type of damage which may have occurred in each case.

The DaDI and the FreDI use, respectively, the relative differences (more specifically, the percent variations) of the damping factors and natural frequencies between a system's reference and damaged states to search for the most likely damage location (equations (1) and (4)). Plots of these relative differences for the three different damage simulations in location A1 are shown in figure 4. 
(a)

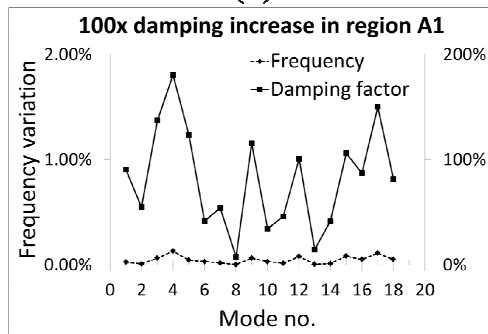

(b)

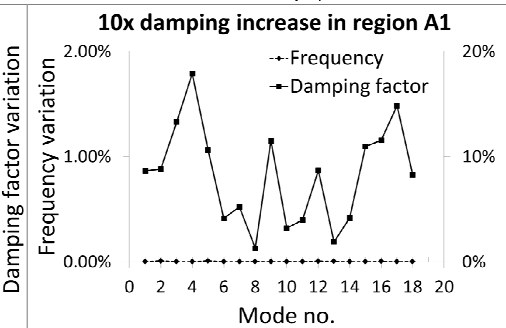

(c)

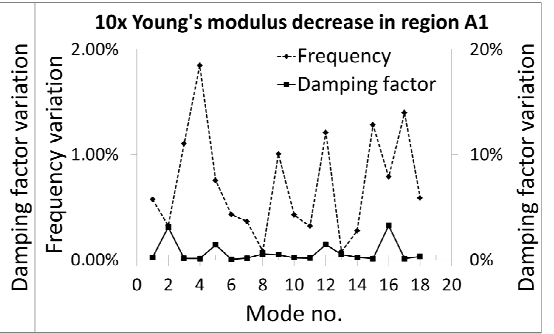

Figure 4. Plots of the relative differences of the damping factors and natural frequencies between a system's reference and damaged states. These plots correspond to three different damage simulations in location A1.

For 100x and 10x local damping increases (cases a and b), 180\% and 17.9\% maximum shifts in the modal damping factors were observed, whereas all the natural frequencies remained practically unchanged (the natural frequencies incurred a slight shift to a maximum of $0.13 \%$ only). For a 10x local Young's modulus reduction (case c), the natural frequencies were shifted as expected, with its maximum value equal to only $1.8 \%$. The modal damping factors also changed reaching a maximum of $3.2 \%$. If the weighting functions had been discarded, the MuDI as shown in figure $\mathbf{3}$ would probably not be as effective in showing the damage location.

\section{Experimental results}

\section{Experimental setups and specimens tested}

Experimental testing was performed on two types of CFRP plates: a quasi-isotropic and an orthotropic plate. The quasi-isotropic specimens are made from a [0/45/135/90 $]_{S}$ layup and approximate dimensions of $392 \times 242 \times 2 \mathrm{~mm}$. Each layer is a woven prepreg constituted by Hexcel® G803 3K 5H satin carbon fiber impregnated in HexPly® 200 phenolique matrix (200/40\%/G803). These specimens are called specimens type A. The orthotropic specimens are made from a $[0 / 90]_{2 \mathrm{~S}}$ layup and have $360 \times 262 \times 3 \mathrm{~mm}$ 
approximate dimensions. Each layer is an epoxy resin impregnated carbon fiber satin weave Cytec ${ }^{\circledR}$ Cycom ${ }^{\circledR}$ 934-37-3KT300 8H (such as that used in some parts of the Lockheed Martin C-130 airplane ${ }^{25}$ ). These specimens are called specimens type B.

Both plates were suspended under a free-free simulated configuration with nylon strings. A shaker with push-rod was used for introducing a single-point excitation at the plates' corner. A signal of the type Multisine was used and the force was measured with a PCB 208C01 type force transducer.

Specimen plate type A responses were measured in the 0 to $400 \mathrm{~Hz}$ frequency range (covering 8 modes) with a dual-channel LDV from Polytec® (interferometer OFV-508 and controller OFV-2802i) and target reflective tape 3M Scotchlite ${ }^{\mathrm{TM}}$. One of the reasons for choosing an LDV is that being a non-contact transducer it does not interfere with the dynamic response of the structure. The responses were measured at 4 different locations, at the edge of the plate in order to acquire 4 Frequency Response Functions (FRFs). A schematic of this experimental setup is shown in figure 5.

Specimen plate type $\mathrm{B}$ responses were measured in the 0 to $600 \mathrm{~Hz}$ frequency range (covering 14 modes) at 3 different locations using Teardrop lightweight accelerometers from PCB, type 352A24, weighing $0.8 \mathrm{~g}$ each only. The reason these accelerometers were used instead of an LDV and a different frequency range was employed is because the tests were made in different laboratories on different occasions. 


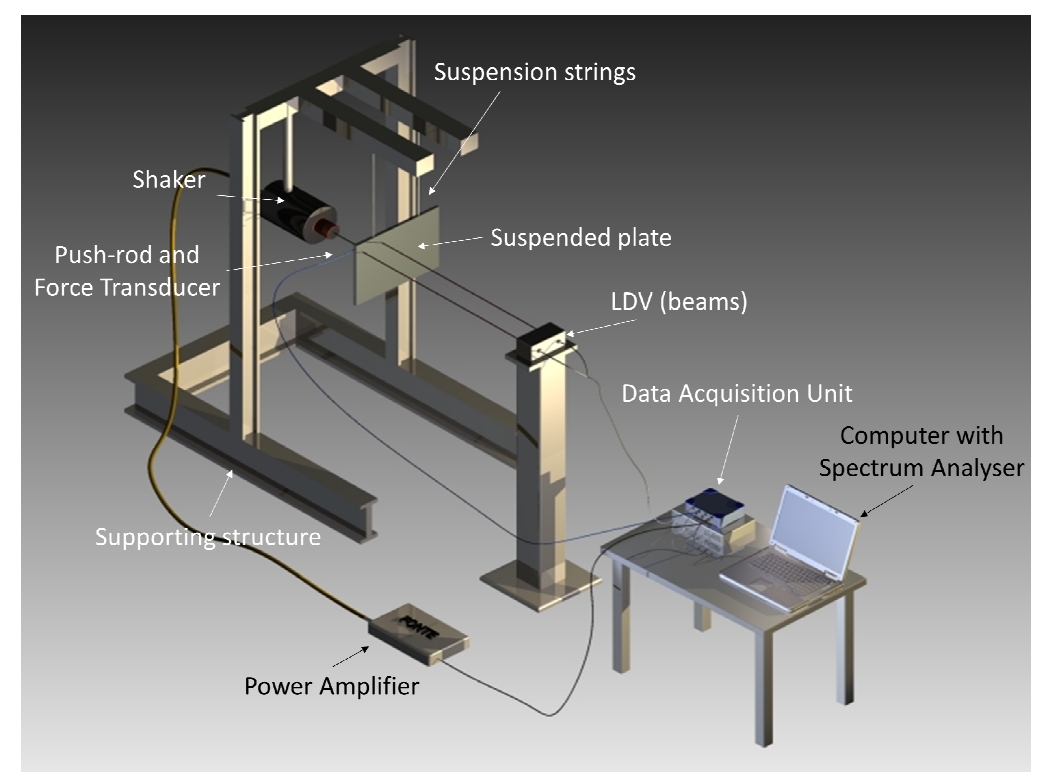

Figure 5. Specimen type $A$ experimental setup.

\section{Damage introduction into the specimens}

Damage was introduced under bending using a quasi-static technique instead of an impact. This was done based on the similitude of impact results with those obtained from quasi-static loading ${ }^{7,28}$ and the process is easier to control. The plates were positioned in a uniaxial test machine between a steel cup, with $80 \mathrm{~mm}$ external diameter and $10 \mathrm{~mm}$ thickness, and a $20 \mathrm{~mm}$ diameter steel hemispherical indenter. Damage was introduced by compressing the plate with the indenter on an undamaged plate and subsequently increased at the same location by increasing the displacement of the indenter sequentially $1 \mathrm{~mm}$ at a time starting at $3 \mathrm{~mm}$. An Instron $10 \mathrm{kN}$ load cell was used to measure the applied force. Because this is a slow test, with velocities set at less than $15 \mathrm{~mm} / \mathrm{min}$, the material's response to damage is not exactly the same as it would be expected from a dynamic impact. Figure 6 shows an example of the LoadDisplacement graph for the three damage cases inflicted on plate A, which areas represent measures of the amount of energy dissipated during the introduction of damage. 


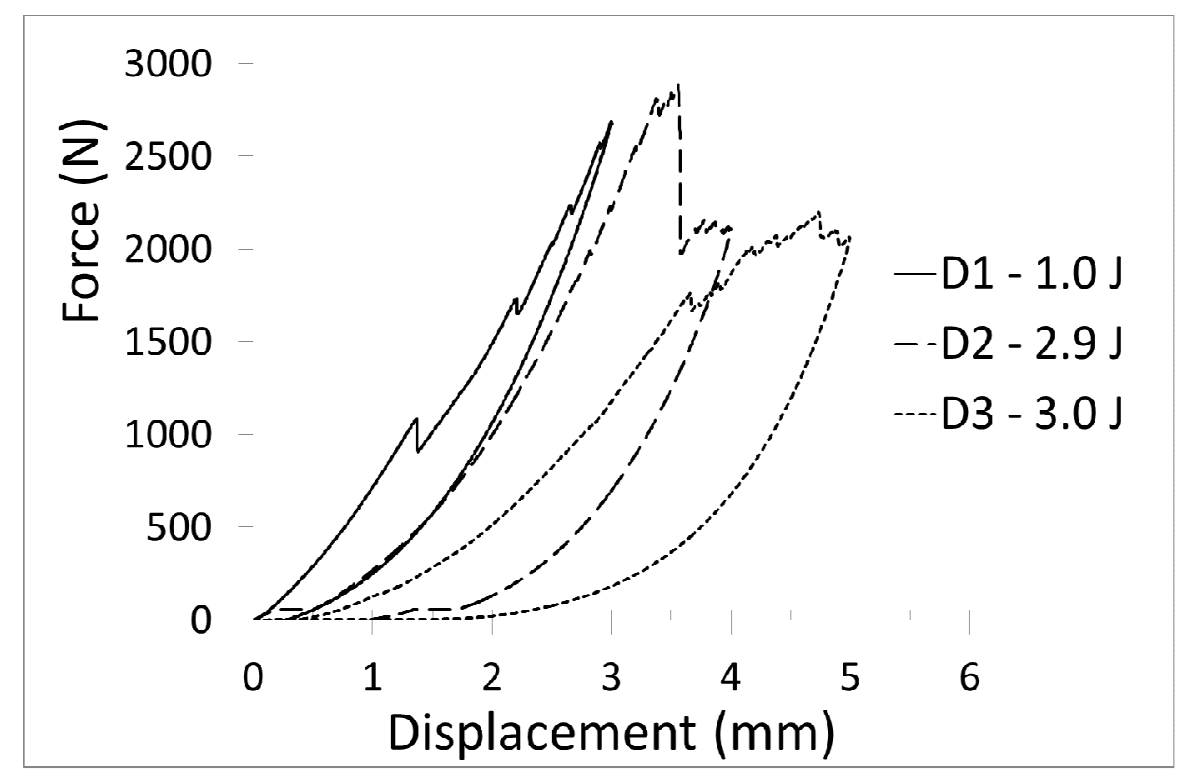

Figure 6. Force-displacement graphs during damage introduction for specimen type $A$.

Damage was inflicted 3 times at the same location. Thus, 4 states are available: healthy (H), damage 1 (D1), damage 2 (D2) and damage 3 (D3). Table 2 summarizes all the damage scenarios (combinations) for both specimen plates type $A$ and $B$.

Table 1. Damage scenarios and energies involved.

\begin{tabular}{lcc}
\hline \multicolumn{1}{c}{ Scenario } & $\begin{array}{c}\text { Specimen A } \\
\text { Energy }(\mathrm{J})\end{array}$ & $\begin{array}{c}\text { Specimen B } \\
\text { Energy }(\mathrm{J})\end{array}$ \\
\hline H-D1 (state D1) & 1.0 & 0.9 \\
H-D2 & 3.9 & 3.8 \\
H-D3 & 6.9 & 5.3 \\
D1-D2 (state D2) & 2.9 & 2.9 \\
D1-D3 & 6.0 & 4.4 \\
D2-D3 (state D3) & 3.0 & 1.5 \\
\hline
\end{tabular}

\section{Evolution of damping and frequency with damage}

The evolution of the experimental modal damping factors and natural frequencies with damage (energy) for both specimens type $A$ and $B$ are plotted in figures 7 and 8 . As expected, these plots suggest that there is a pattern in the way the modal parameters change when damage increase. However, uncertainties associated with the classical 
Experimental Modal Analysis (EMA) and Modal Identification techniques are polluting the data considerably, especially with regards to the Modal Damping Factor ${ }^{25}$.

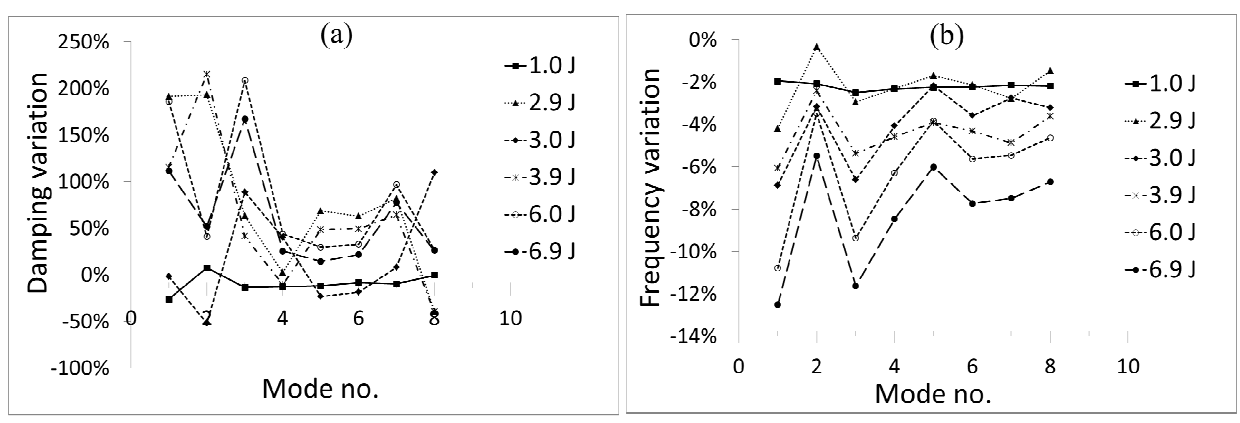

Figure 7. Evolution of the modal damping factors (a) and natural frequencies (b) variations with damage for 8 measured modes concerning specimen type $A$.
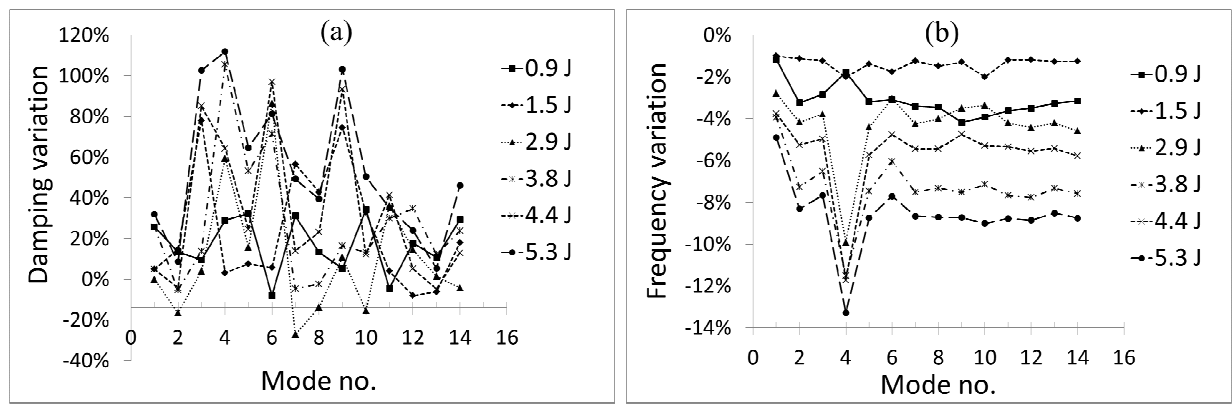

Figure 8. Evolution of modal damping factors (a) and natural frequencies (b) variations with damage for 14 measured modes concerning specimen type $B$.

Another way to assess how the damping and stiffness properties of the composite structure are changing with localized damage is to determine the overall variations (averaged variation values) and plot them with respect to the damage severity (i.e., energy). Results are shown in figures 9 and 10, in which it can be seen that there is a tendency for the overall damping to increase whereas there is a tendency for the overall stiffness to decrease (as expected). While these latter results are not novel, the former is quite relevant, given that the authors are not aware of many other experimental studies 
on the evolution of the modal damping factor with damage in CFRPs, except a few assumptions that point towards that direction ${ }^{17,18,20-25}$.

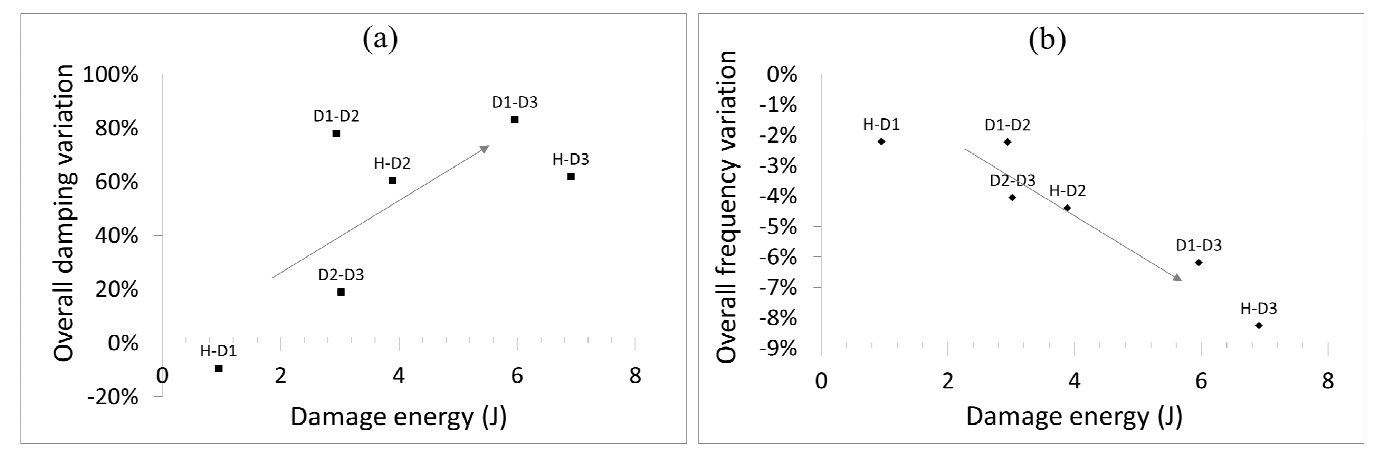

Figure 9. Evolution of the modal damping factors (a) and natural frequencies (b): overall (averaged) variations with damage severity (energy) on specimen type $A$.

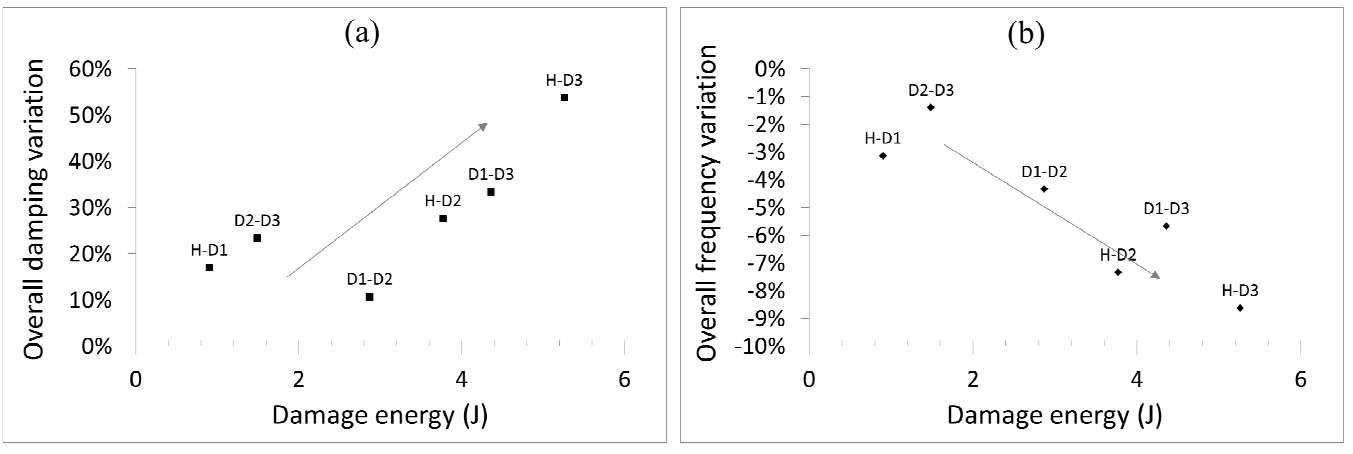

Figure 10. Evolution of the modal damping factors (a) and natural frequencies (b): overall (averaged) variations with damage severity (energy) on specimen type $B$.

\section{Evolution of the MuDI weighting functions with damage}

The previous analysis of the data suggests that the MuDI algorithm has some problems locating the damage, especially for specimen type $B$ in which a larger dispersion of the data points is observed. However, damage location is not the primary aim of this paper and that has already been assessed previously ${ }^{17,18,25}$. In this work, the intent is to assess the sensitivity of the algorithm and the modal parameters - namely the modal damping 
factor - to damage. MuDI's weighting functions, $W_{f}$ and $W_{d}$, are determined as a way of selecting which is the more sensitive feature to the damage location: stiffness or damping. Plots of the frequency weighting function $W_{f}$ with damage severity (energy) are presented in figure 11 (equivalent plots would be obtained for $W_{d}$ from equation (9)).
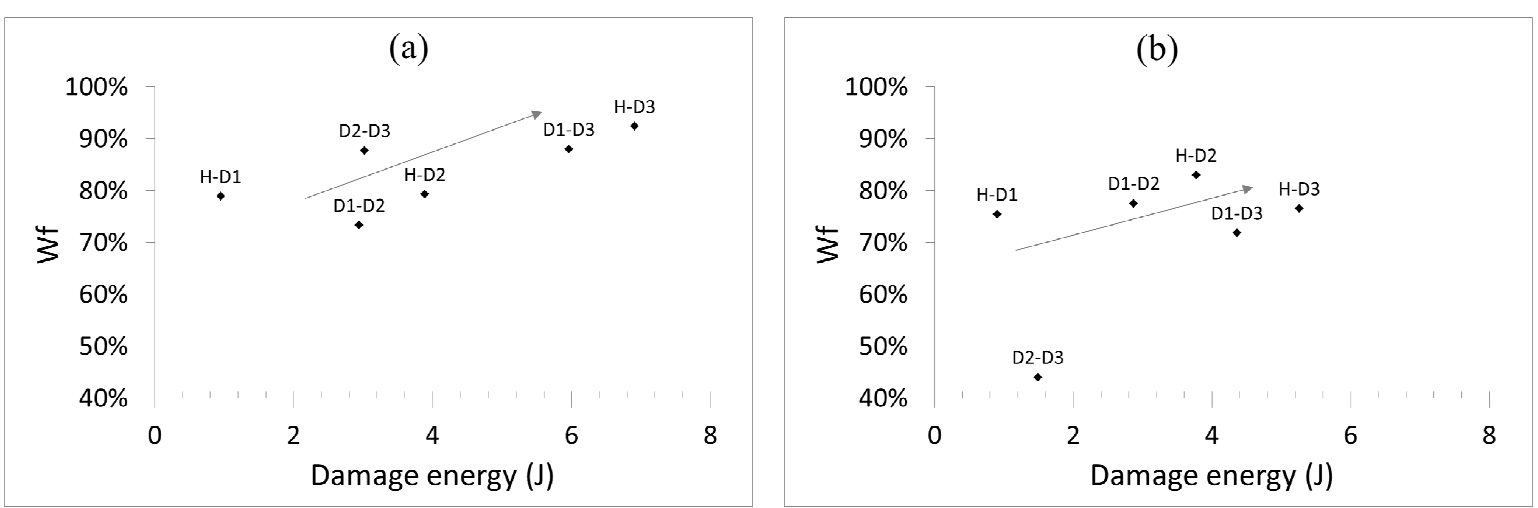

Figure 11. Evolution of the frequency weighting factor $W_{f}$ with damage severity (energy) on specimens type $A$ (a) and $B$ (b).

Figure 11 suggests that the MuDI is being governed by the FreDI index, i.e., by stiffness dependent damage rather than damping. Also, there seems to be a tendency for $W_{f}$ to increase with increasing levels of damage for both specimen plates type $A$ and $B$, although this is more evident for specimen $A$ where the dissipated energy is greater. As an example, two results for the MuDI are presented in figure 12 regarding specimen plate type $A$, in which it can be seen that the $\mathrm{DaDI}$ is not pointing to the correct location accurately. Also, there are a few false-positives, even when the damage location has been correctly identified. 

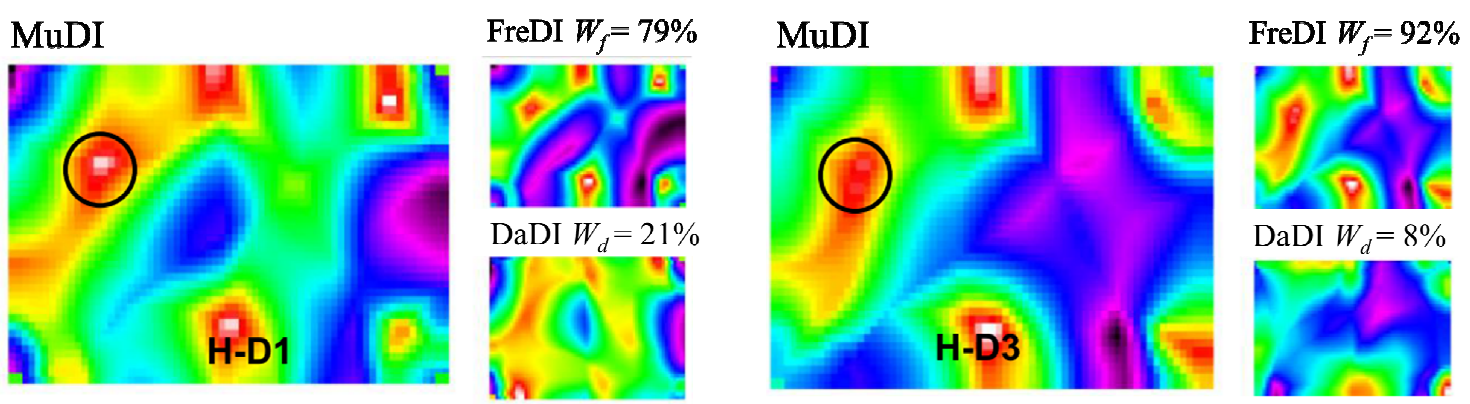

Figure 12. MuDI for damage scenarios H-D1 and H-D3 on specimen plate $A$.

While the algorithm can provide an important indication of the likelihood for damage to be located at a certain region, it is very sensitive to the accurate identification of the modal parameters, namely the modal damping factor. This may be due to concurrent damage types being present at the same time (i.e., fiber breakage, which may lead to a decrease in damping, and debonding, which may lead to an increase in damping), changes introduced by the experimental method itself (e.g., effects from sensors and cables) and the modal identification method itself. This means that the use of algorithms that are based on EMA, such as the MuDI, although usually readily accessible, still have some limitations.

\section{Conclusions}

A study on the understanding of how increasing the level of damage affects the modal damping factor in carbon fiber reinforced laminates is presented. While the influence of damage in stiffness (frequency) is not novel, to the extent of knowledge of the authors there are not many other experimental studies that assess the evolution of the damping factor with increasing damage in CFRPs. Damage was introduced using a quasi-static procedure in two different CFRP laminates. It was quantified from the dissipated energy that was released during its introduction in a complete cycle of loading and unloading. 
Furthermore a modal-based damage location technique that combines both the natural frequencies and the modal damping factors as damage sensitive features was discussed. It was consistently shown, through experimentation, that the modal damping factors tend to increase with real localized damage in CFRPs. Secondly, the use of the dissipated energy as a measure of the damage severity allowed plotting meaningful data relating the modal parameters with increasing level of damage. A similar trend was observed for the overall stiffness variation (as a function of the natural frequencies), although in this case it is a decreasing one with increasing levels of damage.

One of the problems observed is that, although damping is consistently found to increase globally with damage, the determination of the individual changes of the modal damping factors is very uncertain. This may be due to concurrent damage types being present at the same time (i.e., fibre breakage, which may lead to a decrease in damping, and debonding, which may lead to an increase in damping), changes introduced by the experimental method itself (e.g., effects from sensors and cables) and the identification algorithm itself. This means that the use of algorithms such as the MuDI can have some limitations.

Further investigation is still required to better understand if it is possible to find a correlation that may be used effectively to predict with accuracy the level of damage based on the evolution of the modal parameters. This could be an important result towards the development of Prognosis techniques.

\section{Acknowledgments}

This research received no specific grant from any funding agency in the public, commercial, or not-for-profit sectors. 


\section{References}

1 Patterson J. New Large Aircraft Composite Firefighting, http://www.airporttech.tc.faa.gov/safety/bagot2.asp, accessed online on July 2013.

2 Sanders DR, Kim YI and Stubbs N. Nondestructive Evaluation of Damage in Composite Structures Using Modal Parameters, Exp Mech, 32 (3), 240-251, (1992).

3 Jacob PJ, Desforges MJ and Ball AD. Analysis of Suitable Wavelet Coefficients for Identification of the Simulated Failure of Composite Materials, Proceedings of the $2^{\text {nd }}$ International Conference on Structural Damage Assessment using Advanced Signal Processing Procedures (DAMAS 97), Sheffield, England, UK, 31-40, (1997).

4 Matthews FL. Damage in Fiber Reinforced Plastics; Its Nature, Consequences and Detection, Proceedings of the $3^{\text {rd }}$ International Conference on Damage Assessment of Structures (DAMAS 99), Dublin, Ireland, 1-16, (1999).

5 Silva AJPF. Propagação de Delaminagem em Compósitos de Matriz Polimérica, PhD thesis, Instituto Superior Técnico, Technical University of Lisbon, Portugal, (2001).

6 Carvalho RJS. Resistência Residual de um Compósito de Matriz Epóxídica reforçado por Fibras de Carbono após Reparação, $\mathrm{PhD}$ thesis, Instituto Superior Técnico, Technical University of Lisbon, Portugal, (2003).

7 Abrate S. Impact on Composite Structures, Cambridge University Press, (1998).

8 Zak A, Krawczuk M and Ostachowicz W. Vibration of a Laminated Composite Plate with Closing Delamination, Proceedings of the $3^{\text {rd }}$ International Conference on Damage Assessment of Structures (DAMAS 99), Dublin, Ireland, 17-26, (1999). 
Structural Health Monitoring with Special Emphasis on Composite Materials, Shock Vib Dig, 38 (4), 295-324, (2006).

10 Cawley $\mathrm{P}$ and Adams RD. The Location of Defects in Structures from Measurements of Natural Frequencies, J Strain Anal Eng Des, 14 (2), 49-57, (1979).

11 Keye S, Rose M and Sachau D. Localizing Delamination Damages in Aircraft Panels from Modal Damping Parameters, Proceedings of the $19^{\text {th }}$ International Modal Analysis Conference (IMAC XIX), Kissimmee, Florida, USA, 412-417, (2001).

12 Kyriazoglou C, Page BHL and Guild FJ. Vibration Damping for Crack Detection in Composite Laminates, Comp Part A, 35, 945-953, (2004).

13 Yam LH, Wei $\mathrm{Z}$ and Cheng L. Nondestructive Detection of Internal Delamination by Vibration-based Method for Composite Plates, J Comp Mat, 38 (24), 2183-2198, (2004).

14 Zou Y, Tong L and Steven GP. Vibration-Based Model-Dependent Damage (Delamination) Identification and Health Monitoring for Composite Structures - A Review, J Sound Vib, 230 (2), 357-378, (2000).

15 Hadi AS and Ashton JS. Measurement and Theoretical Modeling of the Damping Properties of a Uni-Directional Glass/Epoxy Composite, Compos Struct, 34, 381-385, (1996).

16 Berthelot JM, Assarar M, Sefrani Y and Mahi AE. Damping Analysis of Composite Materials and Structures, Compos Struct, 85, 189-204, (2008).

17 Montalvão D, Ribeiro AMR and Silva JD. A Method for the Localization of Damage in a CFRP Plate using Damping, Mech Syst Signal Pr, 23, 1846-1854, (2009). 
Modal-based Multi-Parameter Method for Locating Damage in Composite Laminates, Exp Mech, 51, 1473-1488, (2011).

19 Rytter A. Vibration Based Inspection of Civil Engineering Structures, PhD thesis, Aalborg University, Denmark, (1993).

20 Tan $\mathrm{CH}$ and Dharan CKH. Cyclic Hysteresis Evolution as a Damage for Notched Composite Laminates, J Comp Mat, 44 (16), 1977-1990, 2010.

21 Idriss M, Mahi AE, Assarar M and Guerjouma RE. Damping Analysis in Cyclic Fatigue Loading of Sandwich Beams with Debonding, Comp Part B, 44, 597-603, 2013. 22 Kyriazoglou C and Guild FJ. Quantifying the Effect of Homogeneous and Localized Damage Mechanisms on the Damping Properties of Damaged GFRP and CFRP Continuous and Woven Composite Laminates - an FEA Approach, Comp Part A, 36, 367-379, 2005 .

23 Kiral Z, Içten BM and Kiral BG. Effect of Impact Failure on the Damping Characteristics of Beam-Like Composite Structures, 43, 3053-3060, 2012.

24 Shahdin A, Mezeix L, Bouvet C, Morlier J and Gourinat Y. Monitoring the Effects of Impact Damages on Modal Parameters in Carbon Fiber Entangled Sandwich Beams, Eng Struct, 31, 2833-2841, 2009.

25 Montalvão D. A Modal-based Contribution to Damage Location in Laminated Composite Plates, $\mathrm{PhD}$ thesis, Instituto Superior Técnico, Technical University of Lisbon, (2010).

26 Maia NMM, Silva JMM and Ribeiro AMR. A New Concept in Modal Analysis: The Characteristic Response Function, Intl J Anal Exp Modal Anal, 9 (3), 191-202, 1994. 

Measurement of the complex Young's modulus on a CFRP laminate considering the constant hysteretic damping model, Compos Struct, 97, 91-98, 2013.

28 Rilo NF, Ferreira LMS and Leal RACP. Low-Velocity Impact Analysis of Glass/Epoxy Plates, Proceedings of the $5^{\text {th }}$ International Conference on Mechanics and Materials in Design (M2D'2006), Porto, Portugal, (2006). 